\title{
LncRNA CDKN2B-AS1 stabilized by IGF2BP3 drives the malignancy of renal clear cell carcinoma through epigenetically activating NUF2 transcription
}

Xina Xie ${ }^{1}$, Jiatian Lin ${ }^{2}$, Xiaoqin Fan ${ }^{3}$, Yuantang Zhong ${ }^{4}$, Yequn Chen ${ }^{5}$, Kaiqing Liu', Yonggang Ren ${ }^{1}$, Xiangling Chen ${ }^{1}$, Daihuan Lai ${ }^{1}$, Xuyi $\mathrm{Li}^{1}$, Zesong $\mathrm{Li}^{1}$ and Aifa Tang $\mathbb{B}^{1}$

\begin{abstract}
Because of the lack of sensitivity to radiotherapy and chemotherapy, therapeutic options for renal clear cell carcinoma (KIRC) are scarce. Long noncoding RNAs (IncRNAs) play crucial roles in the progression of cancer. However, their functional roles and upstream mechanisms in KIRC remain largely unknown. Exploring the functions of potential essential IncRNAs may lead to the discovery of novel targets for the diagnosis and treatment of KIRC. Here, according to the integrated analysis of RNA sequencing and survival data in TCGA-KIRC datasets, cyclin-dependent kinase inhibitor 2B antisense IncRNA (CDKN2B-AS1) was discovered to be the most upregulated among the 14 IncRNAs that were significantly overexpressed in KIRC and related to shorter survival. Functionally, CDKN2B-AS1 depletion suppressed cell proliferation, migration, and invasion both in vitro and in vivo. Mechanistically, CDKN2B-AS1 exerted its oncogenic activity by recruiting the CREB-binding protein and SET and MYND domain-containing 3 epigeneticmodifying complex to the promoter region of Ndc80 kinetochore complex component (NUF2), where it epigenetically activated NUF2 transcription by augmenting local H3K27ac and H3K4me3 modifications. Moreover, we also showed that CDKN2B-AS1 interacted with and was stabilized by insulin-like growth factor 2 mRNA-binding protein 3 (IGF2BP3), an oncofetal protein showing increased levels in KIRC. The Kaplan-Meier method and receiver operating curve analysis revealed that patients whose IGF2BP3, CDKN2B-AS1 and NUF2 are all elevated showed the shortest survival time, and the combined panel (containing IGF2BP3, CDKN2B-AS1, and NUF2) possessed the highest accuracy in discriminating high-risk from low-risk KIRC patients. Thus, we conclude that the stabilization of CDKN2B-AS1 by IGF2BP3 drives the malignancy of KIRC through epigenetically activating NUF2 transcription and that the IGF2BP3/CDKN2B-AS1/NUF2 axis may be an ideal prognostic and diagnostic biomarker and therapeutic target for KIRC.
\end{abstract}

Correspondence: Zesong Li (Izssc@yahoo.com) or

Aifa Tang (tangaifa2018@email.szu.edu.cn)

${ }^{1}$ Guangdong Key Laboratory of Systems Biology and Synthetic Biology for Urogenital Tumors, Shenzhen Second People's Hospital, First Affiliated Hospital of Shenzhen University, 518000 Shenzhen, Guangdong, China

${ }^{2}$ Department of Minimally Invasive Intervention, Peking University Shenzhen Hospital, 518000 Shenzhen, Guangdong, China

Full list of author information is available at the end of the article These authors contributed equally: Xina Xie, Jiatian Lin, Xiaoqin Fan Edited by G. Calin

\section{Introduction}

Renal cell carcinoma (RCC) is the second most common urological malignancy, accounting for $90 \%$ of kidney tumors, of which $\sim 80-90 \%$ are renal clear cell carcinomas $(\text { KIRC })^{1}$. In 2020, there have been nearly 73,750 new RCC cases and 14,830 deaths caused by RCC in the USA ${ }^{2}$. Although localized or locally advanced RCC can be cured by surgical resection, $\sim 30 \%$ of the patients still show local recurrence or distant metastasis within 5 years, and the

\section{(-) The Author(s) 2021}

(c) Open Access This article is licensed under a Creative Commons Attribution 4.0 International License, which permits use, sharing, adaptation, distribution and reproduction cc) in any medium or format, as long as you give appropriate credit to the original author(s) and the source, provide a link to the Creative Commons license, and indicate if changes were made. The images or other third party material in this article are included in the article's Creative Commons license, unless indicated otherwise in a credit line to the material. If material is not included in the article's Creative Commons license and your intended use is not permitted by statutory regulation or exceeds the permitted use, you will need to obtain permission directly from the copyright holder. To view a copy of this license, visit http://creativecommons.org/licenses/by/4.0/. 
5 -year survival rate is only $10 \%$ (refs. ${ }^{3-5}$ ). Owing to the lack of sensitivity to conventional radiotherapy and chemotherapy, immunotherapy and targeted therapy are used as the first-line treatments for KIRC patients with metastasis, but the outcomes are very poor ${ }^{4,6,7}$. This is mainly due to the fact that the etiology and pathogenesis of KIRC have not yet been fully clarified, and clinically effective therapeutic targets for KIRC are very limited. Therefore, it is of utmost importance to improve our understanding of the molecular mechanisms underlying KIRC pathogenesis, and identify novel sensitive and reliable parameters that potentially serve as diagnostic biomarkers and therapeutic targets for the treatment of patients with KIRC ${ }^{8}$.

In 2018, the first RNAi drug (Patisiran) ${ }^{9}$ was approved by the U.S. FDA for the treatment of hereditary transthyretinmediated amyloidosis, further confirming the reliability of nucleic acids as targets for the disease treatment. In fact, nucleic acid drugs, including ribonucleic acid and oligonucleotide drugs, can not only work in cells as conventional small-molecule drugs but can also precisely target the nucleus, achieving effects that traditional drugs cannot. Thus, nucleic acid drugs may help resolve the current lack of targeted drugs for KIRC. Long noncoding RNAs (lncRNAs), a class of RNA molecules that are $>200$ nucleotides in length and have little or no protein-coding function, have been confirmed to be closely related to the tumorigenesis and progression of various cancers ${ }^{10,11}$. Over the past decade, extensive molecular research has shown that some lncRNAs can function as oncogenes and serve as biomarkers for the clinical diagnosis and prognosis of cancer, such as H19, HOTAIR, PVT1, CCAT, UCA1, MALAT1, and XIST ${ }^{12}$. Consequently, lncRNAs are expected to become a promising target of nucleic acid drugs in cancer therapy, and it is important to study the functions of potential essential lncRNAs in controlling cancer initiation and progression in depth.

Therefore, this study aimed to uncover more potential essential lncRNAs that might affect the prognosis of KIRC and identify new diagnostic biomarkers and therapeutic targets for KIRC treatment. In a systematic screen, among the 14 lncRNAs that were significantly overexpressed and related to shorter survival in KIRC, cyclin-dependent kinase inhibitor 2B antisense lncRNA (CDKN2B-AS1) was discovered to be the most upregulated. We then verified the functional roles and molecular process of CDKN2B-AS1 in KIRC malignancy and highlighted its clinical implication. We also demonstrated the upstream mechanism underlying its upregulation in KIRC.

\section{Materials and methods \\ Data mining and analysis}

The differentially expressed lncRNAs between KIRC tissues and normal tissues from RNA-seq in TCGA dataset were analyzed using the circlncRNAnet database (http://120.126.1.61/circlnc/circlncRNAnet/

lncRNA_TCGA/index.php, access date: 2018.02.08, false discovery rate $=0.05$, and a fold-change cutoff $=2)^{13}$. The survival data in TCGA-KIRC dataset were acquired from the OncoLnc website (http://www.oncolnc.org/, access date: 2018.02.08). The RNA expression of CDKN2B-AS1, Ndc80 kinetochore complex component (NUF2), and insulin-like growth factor 2 mRNA-binding protein 3 (IGF2BP3) was profiled based on normalized RNA-seq data in TCGA-KIRC dataset (TCGA_KIRC_exp_HiSeq V2-2015-02-24) from the UCSC Xena Browser (https:// xenabrowser.net/datapages/). The mRNAs co-expressed with CDKN2B-AS1 in TCGA-KIRC cases were identified using the TANRIC database (https://ibl.mdanderson.org/tanric/ _design/basic/query.html, access date: 2019.03.10) ${ }^{14}$ and circlncRNAnet (http://120.126.1.61/circlnc/circlncRNAnet/ lncRNA_TCGA/index.php, access date: 2018.04.19) ${ }^{13}$. The potential target genes of CDKN2B-AS1 were predicted using the LncMAP database (http://bio-bigdata.hrbmu.edu.cn/ LncMAP/, access date: 2018.03.07) ${ }^{15}$. The binding potential of IGF2BPs to CDKN2B-AS1 was analyzed using the RNA-Protein Interaction Prediction (RPISeq) database (http://pridb.gdcb.iastate.edu/RPISeq/).

\section{Collection of KIRC tissues}

A total of 42 pairs of KIRC tissues and matched adjacent normal tissues were obtained from patients admitted to the First Affiliated Hospital of Shantou University Medical College. All subjects provided written informed consent. The study was conducted in accordance with the Declaration of Helsinki, and the protocol was approved by the Ethics Committee of the First Affiliated Hospital of Shantou University Medical College (number: 2020-031). All the tissue samples were obtained directly from surgery after removal of necessary amount of tissue for routine pathology examination and confirmed for KIRC.

\section{Cell culture and treatment}

Human KIRC cell lines 769-P, ACHN, 786-O, Caki-1, and Caki-2 were obtained from the American Type Culture Collection (Rockville, MD, USA). Caki-1 and Caki-2 cells were cultured in McCoy's 5A medium (Gibco, USA), 769-P and 786-O cells were cultured in RPMI-1640 medium (Hyclone, USA), and ACHN cells were cultured in Eagle's MEM medium (Gibco, USA). All media were supplemented with $10 \%$ fetal bovine serum (FBS; Gibco, USA) and all cell lines were cultured in a humidified incubator at $37^{\circ} \mathrm{C}$ with $5 \% \mathrm{CO}_{2}$. The cell lines were authenticated and characterized by the supplier and were monitored regularly for their authenticity (Biowing Applied Biotechnology Co. Ltd, Shanghai, China) and to be free of mycoplasma contamination. 
Small interfering RNAs (siRNAs) and antisense oligonucleotides (ASOs) for CDKN2B-AS1, CREB-binding protein (CBP), SET and MYND domain-containing 3 (SMYD3), and IGF2BP3 were obtained from RiboBio Co. (Guangzhou, China), and were transfected using Lipofectamine RNAiMAX (Invitrogen, USA) according to the manufacturer's instructions. The pcDNA3.1-NUF2 plasmid was obtained from Transheep (Shanghai, China) and transfected at a final concentration of $3.0 \mu \mathrm{g} / \mathrm{mL}$ using FuGENE $^{\circledR}$ HD Reagent (Promega, Madison, WI, USA), according to the manufacturer's instructions. For actinomycin D RNA stability assays, the cells were treated at the indicated time points with $5 \mu \mathrm{g} / \mathrm{mL}$ actinomycin D (Sigma, St Louis, MO, USA). siRNA and ASO primer sequences are specified in Supplementary Table S1.

\section{qRT-PCR}

Total RNA was extracted from tissues and cells using Trizol (Invitrogen, USA), and was reverse-transcribed using a PrimeScript RT reagent Kit with gDNA Eraser (Takara Bio, Inc., Japan) for cDNA synthesis and genomic DNA removal. qRT-PCR was performed using a QuantiNova $^{\text {TM }}$ SYBR Green PCR mix kit (QIAGEN, Germany) and carried out in the Applied Biosystems Prism 7500. The relative expression levels of the analyzed genes were compared to those of $\beta$-actin, and fold changes were calculated using the $2^{-\Delta \Delta \mathrm{Ct}}$ method for cell experiments and the $2^{-\Delta C t}$ method for tissue samples ${ }^{16}$. All qRT-PCR primers used are listed in Supplementary Table S1.

\section{Western blot analysis}

Human KIRC cell lines were lysed in RIPA buffer supplemented with $1 \%$ protease inhibitor cocktail (Millipore, USA). Protein concentrations were determined using a BCA protein assay kit (Beyotime, China). Then, $30 \mu \mathrm{g}$ of the denatured protein samples were subjected to $8 \%$ SDS-PAGE and transferred to a PVDF membrane (Millipore, USA). Immunoblotting involved primary antibodies against NUF2 (1:800; No. 15731-1-AP; Proteintech, USA), CBP (1:1000; No. PA1-847; Thermo Fisher, USA), SMYD3 (1:1000; No. ABE2870; Millipore, USA), IGF2BP3 (1:1000; No. 14642-1-AP; Proteintech, USA), and glyceraldehyde 3-phosphate dehydrogenase (GAPDH; 1:10,000; No. 60004-1-Ig; Proteintech, USA). Corresponding secondary antibodies were applied, and blots were developed using Super ECL Plus Detection Reagent (NovasyGen, China). The intensity of bands was determined with Image $2 \mathrm{X}$ software (National Institutes of Health, Bethesda, MD, USA) and normalized to that of GAPDH.

\section{Cell nuclear and cytoplasmic RNA isolation}

The cytoplasmic and nuclear RNAs of 769-P and ACHN cells were separated using the PARIS kit (Life
Technologies, USA), according to the manufacturer's instructions. The expression levels of CDKN2B-AS1, U3, and GAPDH in the nucleus or cytoplasm were detected by qRT-PCR.

\section{Chromatin immunoprecipitation assay}

As previously described ${ }^{17}$, chromatin immunoprecipitation (ChIP) was performed using EZ-Magna ChIP A and EZ-Magna ChIP G Kits (Millipore, USA), following the manufacturer's instructions. Briefly, ChIP grade antibodies were as follows: anti-histone $\mathrm{H} 3$ lysine 27 acetylation (H3K27ac; Thermo Fisher, USA), anti-histone H3 lysine 4 trimethylation (H3K4me3; Millipore, USA), antihistone $H 3$ lysine 27 trimethylation (H3K27me3; Millipore, USA), anti-P300 (Santa Cruz Biotechnology, USA), anti-CBP (Thermo Fisher, USA), anti-SMYD3 (Millipore, USA), and normal IgG (Millipore, USA). Immunoprecipitated DNA was amplified by qPCR, and normalized to the input DNA. Primer sequences for the promoter region of NUF2 are listed in Supplementary Table S1.

\section{ChIP-seq data analyses}

ChIP-seq data of KIRC samples and normal tissues were obtained from the NCBI Gene Expression Omnibus (GEO) datasets (https://www.ncbi.nlm.nih.gov/gds/). The BED files were downloaded from GEO datasets and were visualized using the WashU EpiGenome Browser (https:// epgg-test.wustl.edu/browser/). The information on ChIPseq data is listed in Supplementary Table S2.

\section{RNA immunoprecipitation assay}

As previously described ${ }^{17}$, the Magna RIP RNA-Binding Protein Immunoprecipitation Kit (Millipore, USA) was used to determine the relationship between CDKN2BAS1 and CBP, SMYD3, or IGF2BP3 according to the manufacturer's instructions. Antibodies used for the RNA immunoprecipitation (RIP) assay were as follows: antiCBP, anti-SMYD3, anti-IGF2BP3, and anti-lgG antibodies ( $5 \mu \mathrm{g}$ per reaction). Coprecipitated RNAs were used for cDNA synthesis and were evaluated by qRT-PCR.

\section{Cell proliferation, colony formation, and 5-ethynyl-2'- deoxyuridine incorporation assays}

The ability of cell was detected using the Cell Counting Kit-8 (CCK-8; Dojindo, Kumamoto, Japan) according to the manufacturer's instructions. A total of $5 \times 10^{3}$ cells/ well were plated into 96-well plates after transfection for $24 \mathrm{~h}$, and $10 \mu \mathrm{L}$ of CCK-8 solution (Dojindo, Japan) was added on days 1,2 , and 3 , respectively. Subsequently, the cells were incubated for $2 \mathrm{~h}$ at $37^{\circ} \mathrm{C}$, and the optical density values were measured at $450 \mathrm{~nm}$ using a microplate reader (Bio-Rad Laboratories, Inc.). For the colony formation assay, transfected cells were seeded in six-well plates at $2 \times 10^{3}$ per well. After $10-14$ days, cell colonies 
were fixed with $4 \%$ paraformaldehyde, air dried, and stained with $0.05 \%$ crystal violet (Beyotime, Shanghai, China). The colonies were imaged and counted. For the 5ethynyl-2'-deoxyuridine (EdU) incorporation assay, transfected cells were seeded into 96-well plates. The EdU incorporation assay kit (RiboBio, China) was used to evaluate cell proliferation according to the manufacturer's instructions. Images were acquired under a fluorescent microscope at $567 \mathrm{~nm}$ excitation.

\section{Wound healing and transwell assays}

Transfected cells were seeded in six-well plates and cultured until $100 \%$ confluence. The cell monolayer was scraped in a straight line using a $10 \mu \mathrm{L}$ pipette tip to create an artificial scratch, washed with PBS twice, and the medium was replaced with serum-free medium. Images were captured at $0,6,12$, and $24 \mathrm{~h}$ for $769-\mathrm{P}$ cells and 0 , 24, 48, and $72 \mathrm{~h}$ for ACHN cells. Cell healing rates were calculated based on the fraction of cell coverage across the line. Cell migration and invasion abilities were measured using transwell chambers $(8-\mu \mathrm{m}$ pore size, BD Biosciences, USA). In the migration assay, $5 \times 10^{4}$ transfected cells cultured in $0.5 \%$ FBS medium were seeded onto the upper chambers of the transwell, and medium with $20 \%$ FBS was added to the lower chamber. After $24 \mathrm{~h}$ of incubation, the chamber was fixed with $4 \%$ paraformaldehyde and stained using $0.05 \%$ crystal violet (Beyotime, Shanghai, China). Cells migrated through the pores were imaged and counted. For the invasion assay, the membrane was precoated with Matrigel and $1.0 \times 10^{5}$ transfected cells were added to the top chamber.

\section{Immunohistochemistry analysis and scoring methods}

Paraffin-embedded KIRC tissue microarrays (No. HkidE180Su02) were purchased from Shanghai Biochip Company Ltd. (Shanghai, China). The sections were dewaxed in xylene and rehydrated in different concentrations of ethanol. Endogenous peroxidase was blocked by $3 \% \mathrm{H}_{2} \mathrm{O}_{2}$ and microwave heating was performed for antigen retrieval. After blocking nonspecific antigen binding with $3 \% \mathrm{BSA}$ at $25^{\circ} \mathrm{C}$ for $1 \mathrm{~h}$, the sections were incubated with a specific primary antibody against IGF2BP3 (1:200; No. 14642-1-AP; Proteintech, USA) at $4{ }^{\circ} \mathrm{C}$ overnight. After incubation with the HRP-conjugated secondary antibody at $37^{\circ} \mathrm{C}$ for $1 \mathrm{~h}$, the sections were counterstained with hematoxylin and stained with diaminobenzidine. Images were taken using a Nikon ECLIPSE Ti microscope (Fukasawa, Japan). The immunostaining intensity of each sample was graded as negative $=0$, weak $=1$, moderate $=2$, or strong $=3$. The proportion of positively stained cells was graded as negative $=0,0-25 \%=1,26-50 \%=2,51-75 \%=3$, or $76-100 \%=4$. The immunostaining score was calculated as the sum of the intensity score and positive rate score.

\section{CDKN2B-AS1 short hairpin RNA and NUF2 overexpression lentiviral vector transduction}

The lentiviral vectors used to overexpress NUF2 and knockdown CDKN2B-AS1 were constructed by OBIO Co. (Shanghai, China) and GenePharma Co. (Shanghai, China), respectively. Human full-length cDNA of NUF2 was cloned into the expression vector pLenti-CMVEGFP-3FLAG-PGK-Zeo, named pLenti-NUF2, and the empty lentiviral expression vector was used as control (pLenti-vector). The short hairpin RNA sequence (5'GGTCATCTCATTGCTCTAT- $\left.3^{\prime}\right)^{18}$ targeting human CDKN2B-AS1 was inserted into the lentiviral pGLV3CMV-EGFP-T2A-Puro vector, designated pGLV3shCDKN2BAS1, and the negative control (NC) was named pGLV3-shNC. ACHN cells were infected for $72 \mathrm{~h}$ with pGLV3-shCDKN2BAS1 or pGLV3-shNC, respectively. Puromycin (Sangon Biotech, China) was used to select stable cell colonies and the stable cell lines were reinfected for $72 \mathrm{~h}$ with pLenti-NUF2 or pLenti-vector, respectively. Stable cell colonies were screened using zeocin (Sangon Biotech, China).

\section{Animal models}

Male BALB/c nude mice (4-6 weeks old) were purchased from Vital River Laboratory Animal Technology Co. (Beijing, China). To detect the effects of CDKN2BAS1 and NUF2 on the tumorigenicity of ACHN cells, ACHN cells $\left(5 \times 10^{6}\right)$ pre-infected with different lentiviral vectors were randomized subcutaneously injected into the flank of BALB/c nude mouse ( $n=6$ or 5 per group). Tumor sizes were measured every 4 days using a Vernier caliper, and the tumor volume was calculated as length $x$ width/2. Mice were sacrificed after injection for 44 days and their tumor tissues were further examined by qRTPCR. Tumor measurements and statistical analysis were performed by researchers who were blinded for the control and treatment groups. All animal experiments were approved by the Animal Experimental Ethics Committee of Shenzhen University.

\section{Statistical analysis}

Statistical analysis was performed using SPSS 23.0 (SPSS Inc., Chicago, IL, USA). The differences between groups for cell and animal experiments were analyzed using an independent sample $t$ test, one-way ANOVA, Student-Newman-Keuls test, or Dunnett multiple comparison test as appropriate. The expression of CDKN2BAS1 in KIRC tissues and matched adjacent normal tissues was analyzed by a paired-sample $t$ test. Survival curves were estimated using the Kaplan-Meier method and compared using log-rank tests. Survival data were evaluated using univariate and multivariate Cox regression analyses. Pearson correlation analysis was used to evaluate the association between different factors. Receiver 
operating curve (ROC) analysis was performed to evaluate the potential of CDKN2B-AS1, NUF2, and IGF2BP3 to differentiate high-risk from low-risk KIRC patients showing area under the curve and 95\% confidence interval (CI). All data are shown as the mean \pm SEM. Statistical significance was defined as $p<0.05$.

\section{Results}

Elevated expression of CDKN2B-AS1 was correlated with poor outcome in patients with KIRC

To identify the important IncRNAs that potentially drove KIRC progression, we detected a total of 1808 upregulated lncRNAs and 512 downregulated lncRNAs in KIRC tissues compared to normal tissues in TCGA datasets, using circlncRNAnet database (Fig. 1a). Meanwhile, we obtained $1558 \operatorname{lncRNAs}$ that were related to survival in KIRC patients from OncoLnc database, and 14 lncRNAs that were both significantly overexpressed and related to a shorter survival (Fig. 1a, b). Of these, CDKN2B-AS1, with a 4.32-log2 [fold change], was the most upregulated lncRNA in KIRC tissues (Fig. 1c). Next, qRT-PCR analysis of CDKN2B-AS1 in 42 pairs of KIRC and matched tumor-adjacent tissues revealed that CDKN2B-AS1 was prominently higher in tumor tissues (Fig. 1d).

Furthermore, TCGA dataset revealed that CDKN2BAS1 was highly expressed in the tissues of patients with a large tumor size, higher tumor grades, advanced tumor node metastasis (TNM) stages, and metastasis (Fig. 1e-h and Supplementary Table S3). Moreover, the Kaplan-Meier survival curve showed that KIRC patients with high CDKN2B-AS1 expression had markedly shorter overall survival (OS), and disease-free survival (DFS) rates than those with low CDKN2B-AS1 expression (Fig. 1i, j). In addition, age, histological grade, TNM stage, tumor invasion, distant metastasis, tumor size, and CDKN2BAS1 levels appeared to be correlated with the survival period of KIRC patients (Supplementary Table S4). The multivariate analysis further demonstrated that CDKN2BAS1 could serve as an independent prognostic factor for worse OS among KIRC patients $(\mathrm{HR}=1.174,95 \% \mathrm{CI}$ : 1.002-1.375, $p=0.047$; Supplementary Table S4). Therefore, our data revealed that high CDKN2B-AS1 expression correlated with poor clinical outcomes in KIRC patients.

\section{Depletion of CDKN2B-AS1 suppresses KIRC growth and metastasis both in vitro and in vivo}

Since CDKN2B-AS1 expression was associated with tumor size and metastasis, we next validated the effects of CDKN2B-AS1 in KIRC cell proliferation and metastasis. Firstly, we found that KIRC cell lines 769-P and ACHN showed higher levels of CDKN2B-AS1 than Caki-1, Caki2, and 786-O cells (Supplementary Fig. S1). Then, we knocked down CDKN2B-AS1 (si-CDKN2B-AS1 mix, including ASO1, ASO3, siRNA1, and siRNA3 against CDKN2B-AS1; Supplementary Fig. S2) in 769-P and ACHN cells, and demonstrated that CDKN2B-AS1 depletion led to the significant suppression of cell viability over a 3-day culture (Fig. 2a, b). Consistently, colony formation assays indicated that CDKN2B-AS1 knockdown significantly reduced the number of colonies formed by $\sim 75 \%$ and $71 \%$ in $769-\mathrm{P}$ and $\mathrm{ACHN}$ cells, respectively, compared with si-NC-transfected cells (Fig. 2c). In addition, wound healing and transwell assays demonstrated that CDKN2B-AS1 suppression also considerably attenuated the migratory and invasive capacities of KIRC cells (Fig. 2d, e).

Moreover, CDKN2B-AS1 expression was decreased in the tumor tissues of mice injected with CDKN2B-AS1depleted ACHN cells (Fig. 2f). As expected, mice in the CDKN2B-AS1-knockdown group exhibited significantly smaller tumor sizes and tumor weights than those in the control group (Fig. 2g-i). Furthermore, we also observed that mice injected with CDKN2B-AS1depleted cells had lower expression of vimentin, matrix metalloprotein 9 (MMP9), matrix metalloprotein 2 (MMP2), AXIN-1, N-cadherin, and higher expression of E-cadherin levels than mice injected with control cells (Fig. 2j). Therefore, our results indicated that CDKN2BAS1 inhibition may be an effective way to diminish KIRC growth and metastasis, thereby decreasing KIRC malignancy in vitro and in vivo.

\section{CDKN2B-AS1 contributes to KIRC progression via NUF2}

To further explore the molecular mechanism of how lncRNA CDKN2B-AS1 contributes to the progression phenotype of KIRC, 718 and 2306 mRNAs co-expressed with CDKN2B-AS1 in KIRC were identified using the TANRIC database and the circlncRNAnet database, respectively, and 6055 mRNAs, related to KIRC survival were obtained from the OncoLnc database. Next, 270 mRNAs, co-expressed with CDKN2B-AS1 and affecting KIRC prognosis, were screened out (Supplementary Fig. S3). Meanwhile, using the LncMAP database, we obtained 54 genes that may be directly regulated by CDKN2B-AS1. By intersection with the above 270 mRNAs, we screened out four potential target genes of CDKN2B-AS1: NUF2, MICALL2, MICAL1, and AGBL2 (Fig. 3a). Interestingly, the qRT-PCR analysis showed that CDKN2B-AS1 knockdown for $48 \mathrm{~h}$ sharply reduced NUF2 mRNA expression, but did not affect MICALL2 or MICAL1 expression in 769-P and ACHN cells (Fig. 3b). In addition, CDKN2B-AS1 depletion significantly increased AGBL2 mRNA expression in ACHN cells, whereas, this effect was not observed in 769-P cells (Fig. 3b). Furthermore, in line with the change in mRNA expression, the depletion of CDKN2B-AS1 also decreased NUF2 protein 
a

\begin{tabular}{|c|c|c|}
\hline \multicolumn{2}{|c|}{ circIncRNAnet (IncRNA -TCGA)* } & \multirow{2}{*}{$\begin{array}{c}\text { OncoLnc (IncRNA-TCGA) }{ }^{8} \\
\text { Survival-related } \\
\text { IncRNAs }\end{array}$} \\
\hline $\begin{array}{l}\text { Up-expression } \\
\text { IncRNAs }\end{array}$ & $\begin{array}{l}\text { Down-expression } \\
\text { IncRNAs }\end{array}$ & \\
\hline 1808 & 512 & 1558 \\
\hline
\end{tabular}

C

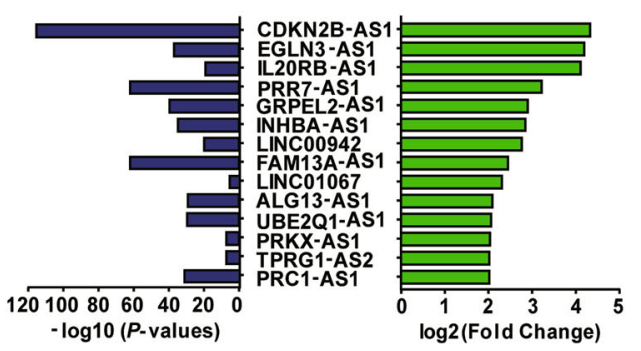

e

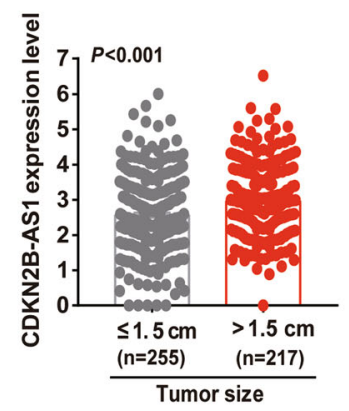

h

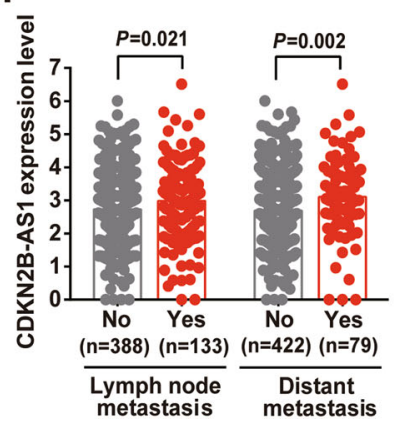

f

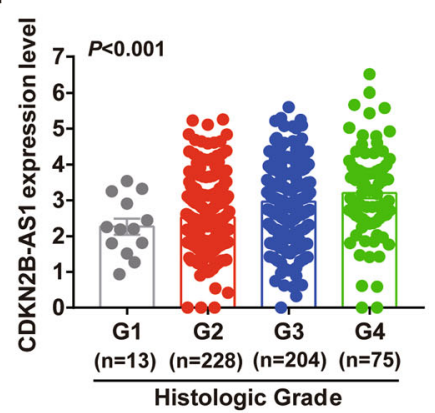

i

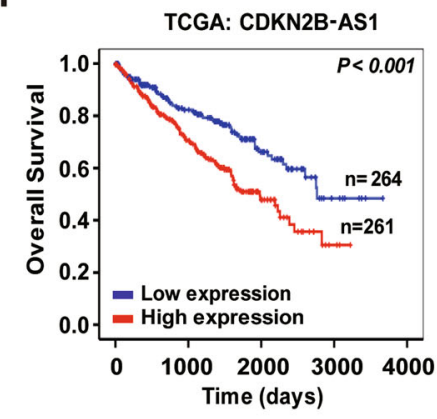

b

circlncRNAnet: up-expression IncRNAs

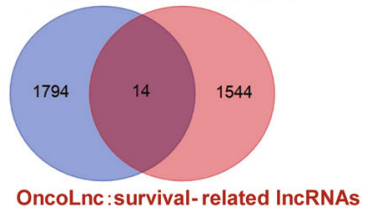

d

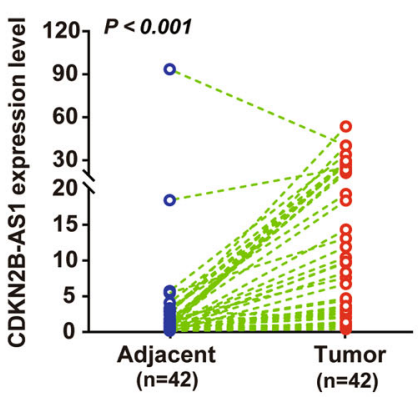

g

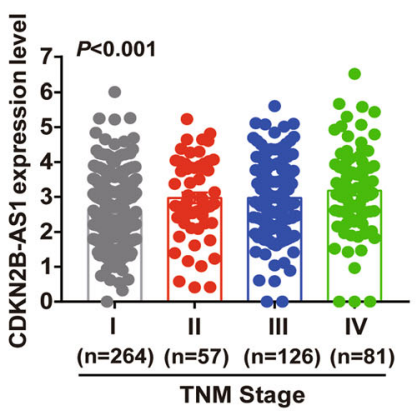

j

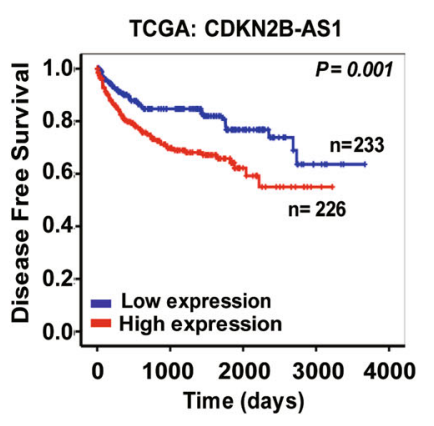

Fig. 1 Identification of an essential IncRNA CDKN2B-AS1 whose overexpression is associated with poor prognosis of KIRC. a Analysis of InCRNAs which were differentially expressed and related to KIRC survival in TCGA, using circlncRNAnet and OncoLnc database, respectively. $\mathbf{b}$ Venn diagram presenting the IncRNAs that were upregulated (1808 IncRNAs), related to survival (1558 IncRNAs), or both (14 IncRNAs) in KIRC. c The 14 IncRNAs which were upregulated and related to a shorter survival in KIRC. $\mathbf{d}$ qRT-PCR analysis of CDKN2B-AS1 expression in 42 paired KIRC tissues (tumor) and adjacent normal tissues (adjacent); $\beta$-actin was used as the internal control. e-h CDKN2B-AS1 expression was analyzed in KIRC patients regarding tumor size $(\mathbf{e})$, histologic grade $(\mathbf{f})$, TNM stage $(\mathbf{g})$, and metastasis status $(\mathbf{h})$, using RNA-seq data in TCGA. Error bars represent the SEM. $\mathbf{i}$, j Kaplan-Meier analysis showed the overall survival and disease-free survival in KIRC patients based on the expression of CDKN2B-AS1 in TCGA dataset, the high and low expression groups were divided by the median of CDKN2B-AS1 levels. $P$ value calculated by paired-sample $t$ test $(\mathbf{d})$, independent sample $t$ test $(\mathbf{e}, \mathbf{h})$, one-way ANOVA $(\mathbf{f}, \mathbf{g})$, or log-rank test $(\mathbf{i}, \mathbf{j})$.

levels (Fig. 3c). Therefore, we speculated that NUF2 may be one of the target genes of CDKN2B-AS1, and play a role in CDKN2B-AS1-mediated KIRC progression.
Subsequently, to evaluate the potential role of NUF2 in CDKN2B-AS1-mediated KIRC progression, KIRC cells were overexpressed NUF2 following CDKN2B-AS1 knockdown. 


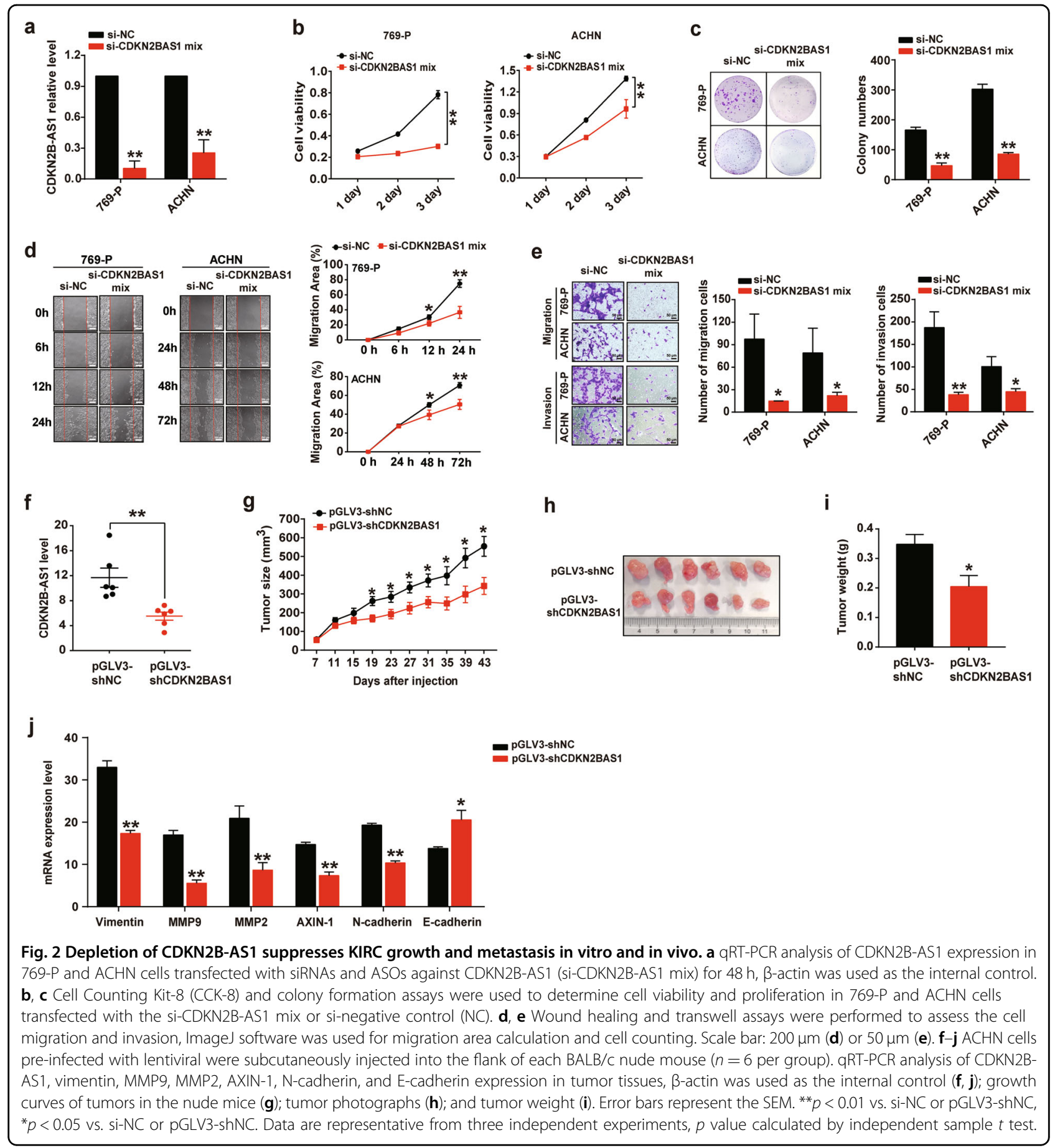

As shown in Fig. 3d-g, the restoration of NUF2 expression significantly ameliorated the inhibitory effects of CDKN2BAS1 depletion on cell proliferation, migration, and invasion in vitro. Consistently, results of rescue experiments showed that ectopic NUF2 expression in CDKN2B-AS1-depleted ACHN cells could significantly recover the tumorigenicity and metastatic potential in vivo. Mice in this group showed similar tumor sizes and weights, and according expression level of epithelial-mesenchymal transition molecules, including vimentin, MMP9, MMP2, AXIN-1, N-cadherin, and E-cadherin, compared to mice in the control group (Fig. 3h-l). Thus, our in vitro and in vivo data confirmed that CDKN2B-AS1 is involved in KIRC progression at least partly through the regulation of NUF2, suggesting that the CDKN2B-AS1/NUF2 axis is important for the regulation of KIRC tumor growth and metastasis. 


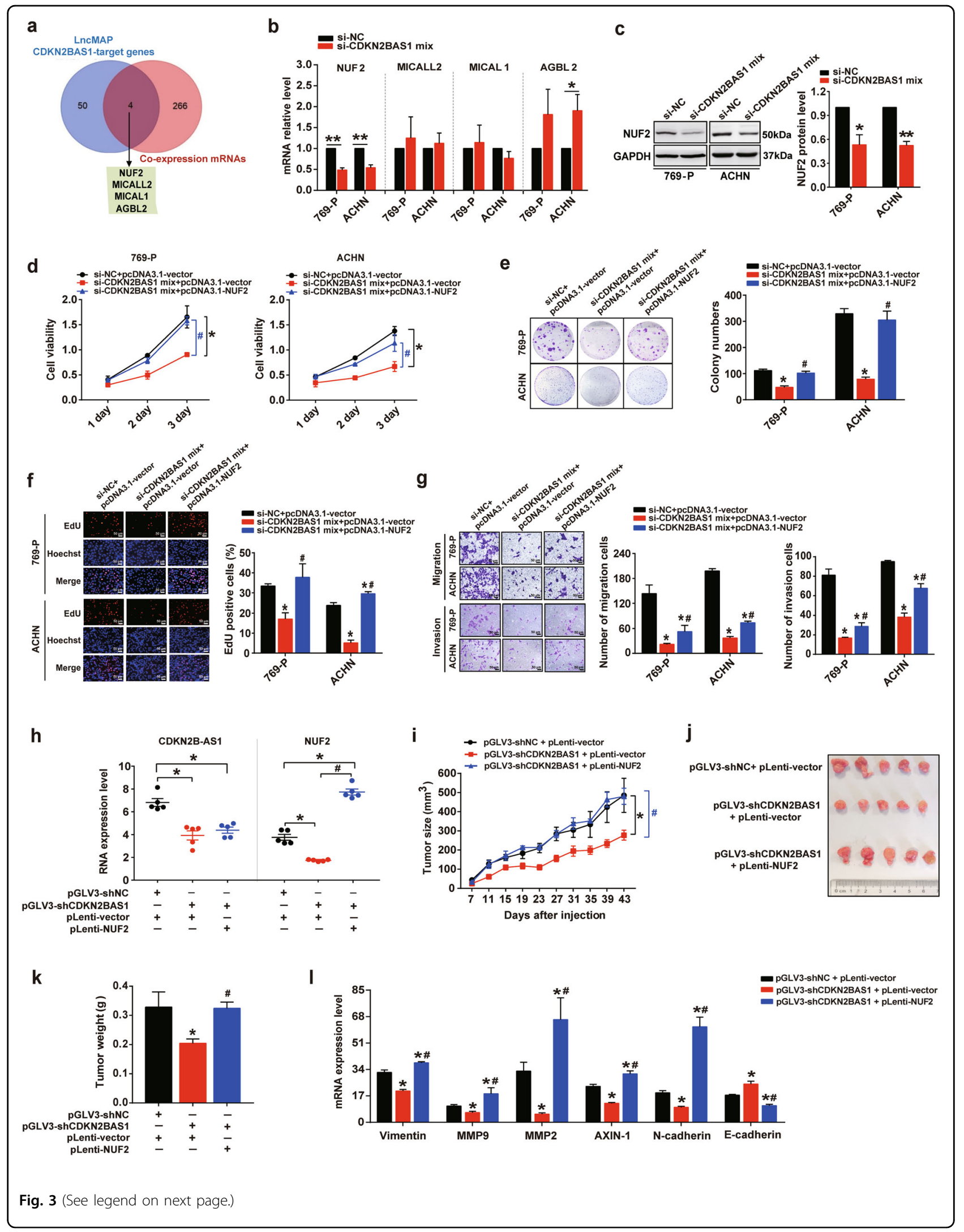


(see figure on previous page)

Fig. 3 CDKN2B-AS1 contributes to KIRC progression via NUF2 in vitro and in vivo. a Venn diagram showing the genes that may be regulated by CDKN2B-AS1 (54 genes), co-expressed with CDKN2B-AS1 (270 genes), or both (4 genes). b, c qRT-PCR analysis of NUF2, MICALL2, MICAL1, and AGBL2 expression in 769-P and ACHN cells transfected with the si-CDKN2B-AS1 mix for $48 \mathrm{~h}$, $\beta$-actin was used as the internal control (b); western blot analysis of NUF2 protein expression with GAPDH as the internal control and quantitation of protein levels performed using densitometry (c). d-g 769-P and ACHN cells were transfected with si-CDKN2B-AS1 mix plus pcDNA3.1-NUF2 plasmid for 48 h; CCK-8 (d), colony formation (e), and EdU (f) assays were performed to assess cell viability and proliferation; transwell assays (g) were used to evaluate cell migration and invasion; Image J software was used for cell counting. Scale bar: $50 \mu \mathrm{m}$. h-I qRT-PCR analysis of CDKN2B-AS1, NUF2, vimentin, MMP9, MMP2, AXIN-1, N-cadherin, and E-cadherin expression in tumor tissues of BALB/c nude mice subcutaneously injected in the flank with ACHN cells pre-infected with the lentiviral vector ( $n=5$ per group); $\beta$-actin was used as the internal control $(\mathbf{h}, \mathbf{l})$; growth curves of tumors in the nude mice $(\mathbf{i})$; representative images $(\mathbf{j})$, and weight of the xenografts derived from the nude mice (k). Error bars represent the SEM, ${ }^{* *} p<0.01$ vs. si-NC; ${ }^{*} p<0.05$ vs. si-NC or si-NC + pcDNA3.1vector or pGLV3-shNC + pLenti-vector; ${ }^{\#} p<0.05$ vs. si-CDKN2B-AS1 mix + pcDNA3.1-vector or pGLV3-shCDKN2BAS1 + pLenti-vector. Data are representative from at least three independent experiments, $p$ value calculated by independent sample $t$ test $(\mathbf{b}, \mathbf{c})$, one-way ANOVA and Student-Newman-Keuls test $(\mathbf{d}-i, \mathbf{k}, \mathbf{l})$.

\section{CDKN2B-AS1 regulates NUF2 transcription by recruiting the CBP and SMYD3 epigenetic modification complex to the promoter region}

Next, we examined the underlying mechanism of NUF2 regulation by CDKN2B-AS1 in KIRC. Given that the distribution of lncRNAs generally determines their function, we first performed subcellular fractionation assays to study the location of CDKN2B-AS1 in KIRC cells. CDKN2B-AS1 expression was 2.13- and 3.12-fold more abundant in the nuclear fraction compared to the cytoplasm in 769-P and ACHN cells, respectively, implying that CDKN2B-AS1 might modulate NUF2 expression during transcriptional processing (Fig. 4a). In addition, ChIP-seq data downloaded from GEO datasets showed higher H3K27ac and H3K4me3 enrichment at the NUF2 promoter region in KIRC tissues and tumor tissue-derived primary cells compared with normal renal tissues (Fig. 4b). This was underscored by ChIP experimental findings showing $\mathrm{H} 3 \mathrm{~K} 27 \mathrm{ac}$ and $\mathrm{H} 3 \mathrm{~K} 4 \mathrm{me} 3$ enrichment at the NUF2 promoter in 769-P and ACHN cells (Fig. 4c). These results could explain why NUF2 expression was increased in KIRC tissues and suggest that it may be regulated by histone epigenetic modification. Thus, we hypothesized that CDKN2B-AS1 may induce histone epigenetic alterations that regulate NUF2 expression in KIRC.

To verify the above hypothesis, ChIP assays were performed and we observed $\sim 75 \%$ and $42 \%$ decrease in H3K27ac and H3K4me3 levels, respectively, in the NUF2 promoter after CDKN2B-AS1 knockdown for $48 \mathrm{~h}$ in 769P cells; however, the H3K27me3 levels showed no significant changes (Fig. 4d). Correspondingly, CDKN2BAS1 knockdown resulted in a $52 \%$ and $68 \%$ decrease in histone acetyltransferase $\mathrm{CBP}$ and methyltransferase SMYD3 recruitment, respectively, in the NUF2 promoter region in 769-P cells, but did not affect acetyltransferase P300 (Fig. 4e). The effects were also confirmed in the ACHN cells (Fig. 4d, e). Furthermore, we found that CBP and SMYD3 enriched in the NUF2 promoter, and the depletion of CBP and SMYD3 by siRNA both dramatically decreased the mRNA and protein levels of NUF2 in 769-P and ACHN cells (Fig. 4f-h). RIP assay showed an $\sim 49-$ and 22-fold CDKN2B-AS1 enrichment in the anti-CBP and anti-SMYD3 immunoprecipitates in the 769-P cells, respectively, compared to the IgG control (Fig. 4i). Similar results were observed in the ACHN cells. Therefore, we concluded that CDKN2B-AS1 stimulated NUF2 transcription by interacting with the CBP and SMYD3 proteins, thereby recruiting this epigenetic modification complex to alter H3K27ac and H3K4me3 enrichment in the NUF2 promoter region.

\section{CDKN2B-AS1 is stabilized by IGF2BP3 in KIRC}

Although most studies on the mechanism underlying CDKN2B-AS1 regulation have mainly investigated at the transcriptional level ${ }^{19}$, little is known about the regulation of RNA stability, which could be affected by RNA-binding proteins. IGF2BPs, classic RNA-binding proteins, have been proven to affect the RNA stability of target genes and participate in the progression of various tumors ${ }^{20}$. Intriguingly, analysis of the binding potential of IGF2BPs to CDKN2B-AS1 on the RPISeq database showed that the Random Forest classifier and Support Vector Machine classifier values were both $>0.5$, implying possible binding between IGF2BP1, IGF2BP2, or IGF2BP3 and CDKN2BAS1 (Supplementary Fig. S4a). Therefore, we hypothesized that the IGF2BP protein family might interact with CDKN2B-AS1 and affect its stability in KIRC.

Thus, we next sought to elucidate whether IGF2BPs could regulate the stability of CDKN2B-AS1. RNA-seq data from TCGA datasets indicated that IGF2BP2 expression in KIRC tissue was decreased and IGF2BP3 expression increased, whereas there was no significant change regarding IGF2BP1 (Supplementary Fig. S4,b). Therefore, only the change in IGF2BP3 was consistent with that of CDKN2B-AS1 in KIRC. This was confirmed by the higher immunoreactive score of IGF2BP3 staining in KIRC tissue compared with normal tissue (Supplementary Fig. S4c, d), and a significant positive correlation 
a

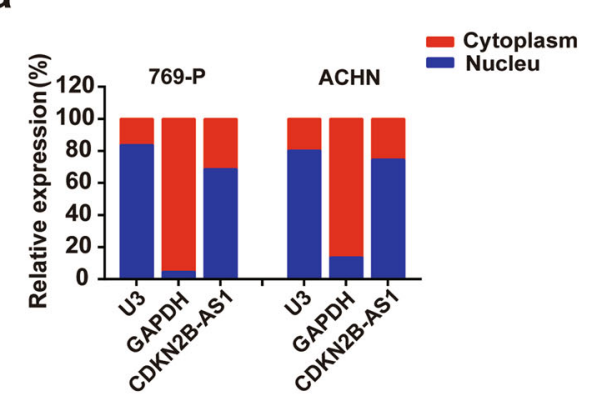

b

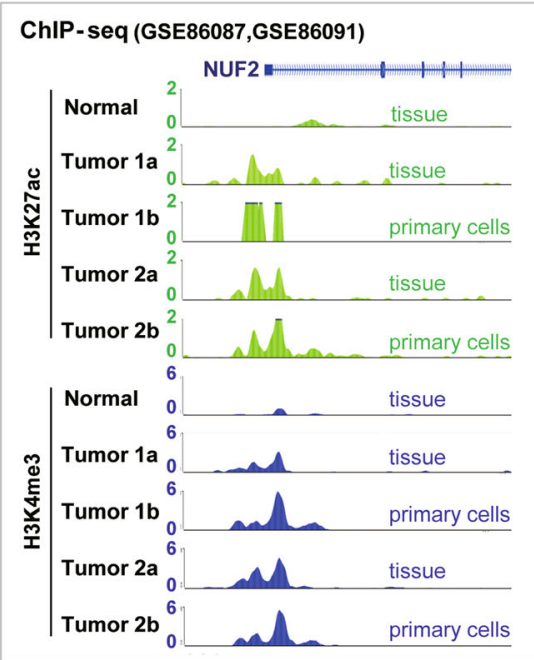

f

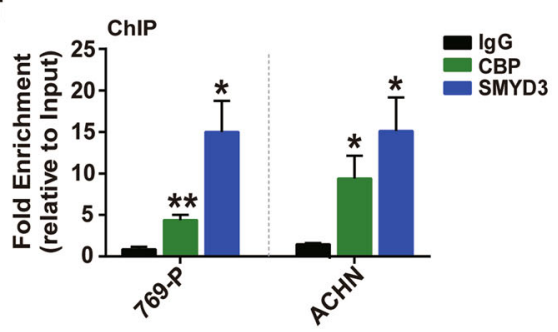

C

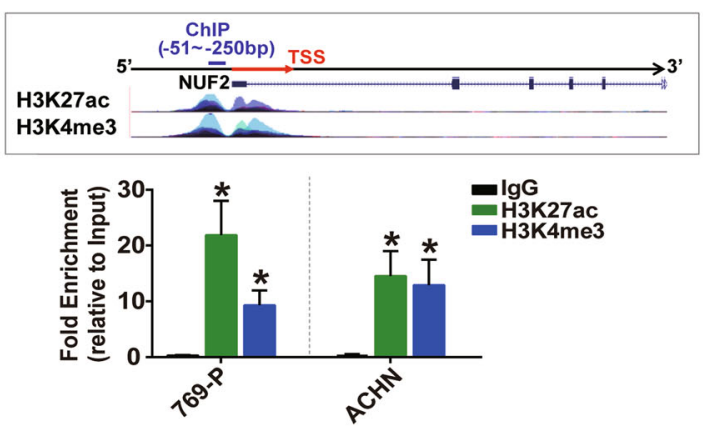

d

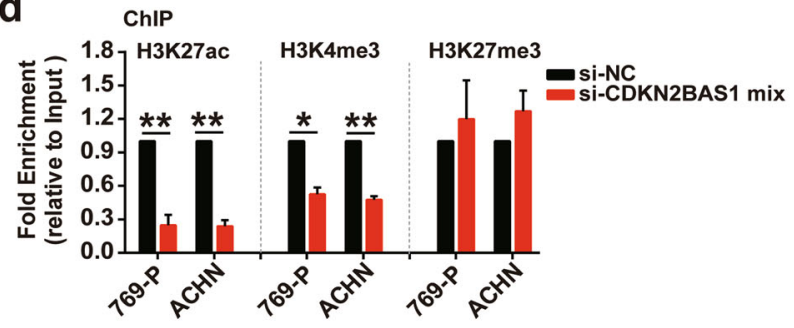

e

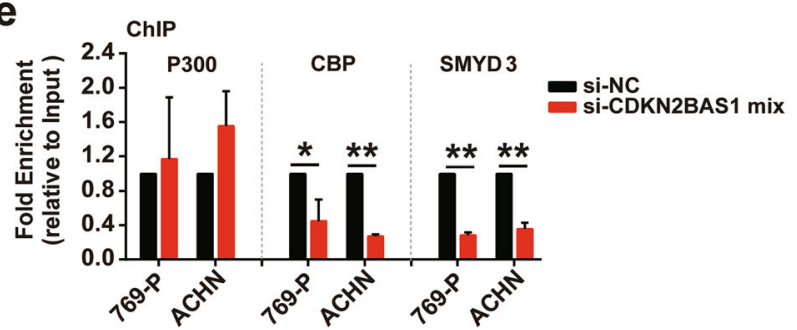

g

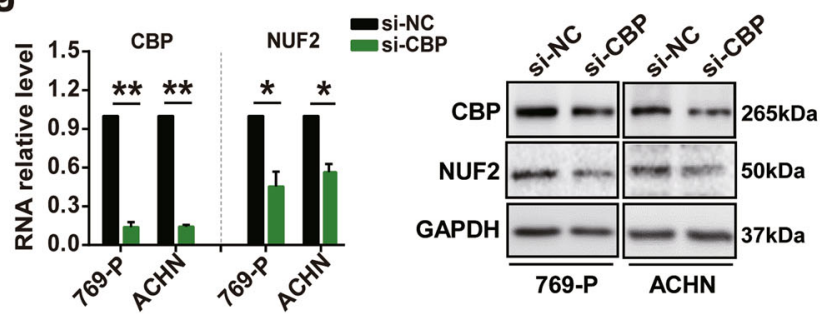

h

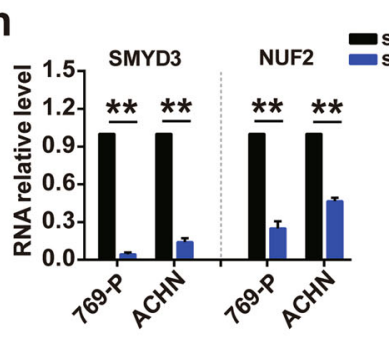

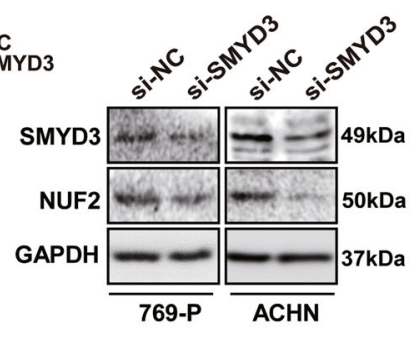

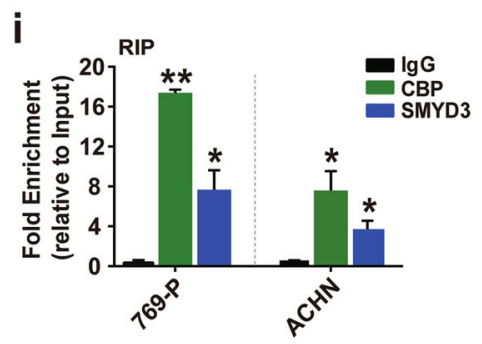

Fig. 4 (See legend on next page.) 
(see figure on previous page)

Fig. 4 CDKN2B-AS1 regulates NUF2 transcription by acting as a modular scaffold of CBP and SMYD3 complex to affect H3K27ac and H3K4me3 modification. a qRT-PCR subcellular analysis of CDKN2B-AS1 in 769-P and ACHN cells, U3 and GAPDH acted as nucleus and cytoplasm markers, respectively. $\mathbf{b}$ ChIP-seq analysis of H3K27ac and H3K4me3 enrichment at the NUF2 promoter region in normal renal tissues, KIRC tissues, and tumor tissue-derived primary cells. $\mathbf{c}$, f ChIP analysis of H3K27ac and H3K4me3 (c), and the chromatin-modifying enzymes CBP and SMYD3 (f) enrichment in the NUF2 gene promoter; ChIP enrichment was measured using real-time PCR, normalized by the input DNA. d, e 769-P and ACHN cells were transfected with the si-CDKN2B-AS1 mix for 48 h; ChIP analysis of H3K27ac, H3K4me3, and H3K27me3 (d), and the chromatin-modifying enzymes P300, CBP, and SMYD3 (e) enrichment in the NUF2 gene promoter; ChIP enrichment was measured using real-time PCR, normalized by the input DNA. g, h qRT-PCR and western blot analysis of CBP, SMYD3, and NUF2 mRNA and protein expression in 769-P and ACHN cells transfected with $\operatorname{si-CBP}(\mathbf{g})$ and si-SMYD3 $(\mathbf{h})$ for $48 \mathrm{~h}$, respectively; $\beta$-actin or GAPDH was used as the internal control, respectively. i RIP assay analysis of CBP and SMYD3 enrichment in CDKN2B-AS1 in 769-P and ACHN cells; RIP enrichment was measured using qRT-PCR normalized by the input RNA. Error bars represent the SEM from three independent experiments, ${ }^{* *} p<0.01$ vs. IgG or si-NC, ${ }^{*} p<0.05$ vs. IgG or si-NC. $P$ value calculated by independent sample $t$ test $(\mathbf{d}, \mathbf{e}, \mathbf{g}, \mathbf{h})$, one-way ANOVA, and Dunnett multiple comparison test $(\mathbf{c}, \mathbf{f}, \mathbf{i})$. TSS transcription start site.

was noted between CDKN2B-AS1 expression and IGF2BP3 levels (Fig. 5a). Furthermore, we noticed that knockdown of IGF2BP3 observably reduced CDKN2BAS1 and NUF2 expression in 769-P and ACHN cells (Fig. 5b, c). Interestingly, si-IGF2BP3 decreased the levels of the remaining CDKN2B-AS1 by $\sim 58.3 \%$ and $54.5 \%$ after actinomycin D $(5 \mu \mathrm{g} / \mathrm{mL})$ treatment for 12 and $24 \mathrm{~h}$, respectively (Fig. 5d). Similarly, we confirmed that IGF2BP3 affected CDKN2B-AS1 stability in ACHN cells (Fig. 5d). However, silencing IGF2BP3 did not affect NUF2 stability (Fig. 5e), indicating that the regulation of NUF2 expression by IGF2BP3 was carried out through the stabilization of CDKN2B-AS1. Accordingly, the RIP assay detected that the anti-IGF2BP3 antibody could dramatically enrich of CDKN2B-AS1 and another known IGF2BP3targeting lncRNA (linc01138) ${ }^{21}$, but not of NUF2 (Fig. 5f). Together, our data demonstrate that IGF2BP3 interacted with and stabilized CDKN2B-AS1 in KIRC cells.

Meanwhile, the depletion of IGF2BP3 prominently inhibited KIRC cell proliferation, migration, and invasion (Fig. 5g, h). Elevated expression of IGF2BP3 was correlated with poor outcome and it could be an independent prognostic factor for worse OS in KIRC patients (Fig. 5i-1 and Supplementary Tables S3-S5). Overall, IGF2BP3 may serve as an oncogene that mediates the KIRC malignant progression, similar to CDKN2B-AS1.

IGF2BP3/CDKN2B-AS1/NUF2 axis is an attractive candidate as a KIRC prognostic and diagnostic biomarker

Based on the mechanisms described above, we proceeded to explore the prognostic and diagnostic potential of the IGF2BP3/CDKN2B-AS1/NUF2 axis in KIRC patients. Pearson correlation analysis from TCGA dataset and the clinical patient cohort showed that the levels of IGF2BP3, CDKN2B-AS1, and NUF2 were significantly positively correlated with each other in KIRC tissue (Fig. 6a-c), concordant with the above findings. Subsequently, we generated a new panel containing IGF2BP3, CDKN2B-AS1, and NUF2 to predict KIRC prognosis. Kaplan-Meier survival analysis and the log-rank test suggested that the OS and DFS gradually decreased with the increase in the number of upregulated markers, and patients with three upregulated markers showed the shortest duration of OS and DFS (Fig. 6d, e). Cox regression multivariate analysis illustrated that the number of upregulated markers could serve as an attractive predictor to evaluate the prognosis of KIRC patients (Table 1). Consistently, in the ROC analysis, the combined panel (containing IGF2BP3, CDKN2B-AS1, and NUF2) showed a higher predictive value for OS and DFS compared to any individual marker (Fig. 6f, g and Supplementary Table S6).

Moreover, ROC analysis was also performed to evaluate the diagnostic accuracy of the IGF2BP3/CDKN2B-AS1/ NUF2 axis for differentiating the clinical characteristics of KIRC patients. As expected, the combined panel showed the highest accuracy in discriminating high tumor grades (G3 and G4) from low tumor grades (G1 and G2), advanced stages (stages III and IV) from early stages (stages I and II), distant metastasis from no distant metastasis, and lymph node metastasis from no lymph node metastasis (Fig. $6 \mathrm{~h}-\mathrm{k}$ and Supplementary Table S6). These results indicate that the IGF2BP3/CDKN2B-AS1/ NUF2 axis can be used as a potential diagnostic parameter to distinguish high-risk from low-risk KIRC patients.

\section{Discussion}

Accumulating evidence has demonstrated that CDKN2B-AS1 is upregulated in a variety of human cancers, such as bladder, gastric, and breast cancers, and serves as an oncogene that plays important roles in tumor progression $^{19}$. Although two other studies have investigated the role of CDKN2B-AS1 in RCC and found as well that it is overexpressed ${ }^{22,23}$, they were limited to verify the effects of CDKN2B-AS1 in vitro experiments, and did not discuss the reasons for the upregulation of it. In the present study, we demonstrated that CDKN2B-AS1 was elevated and correlated with poor clinical outcomes in KIRC, and promoted cell proliferation, migration, and invasion in vitro. In addition, we further verified the 


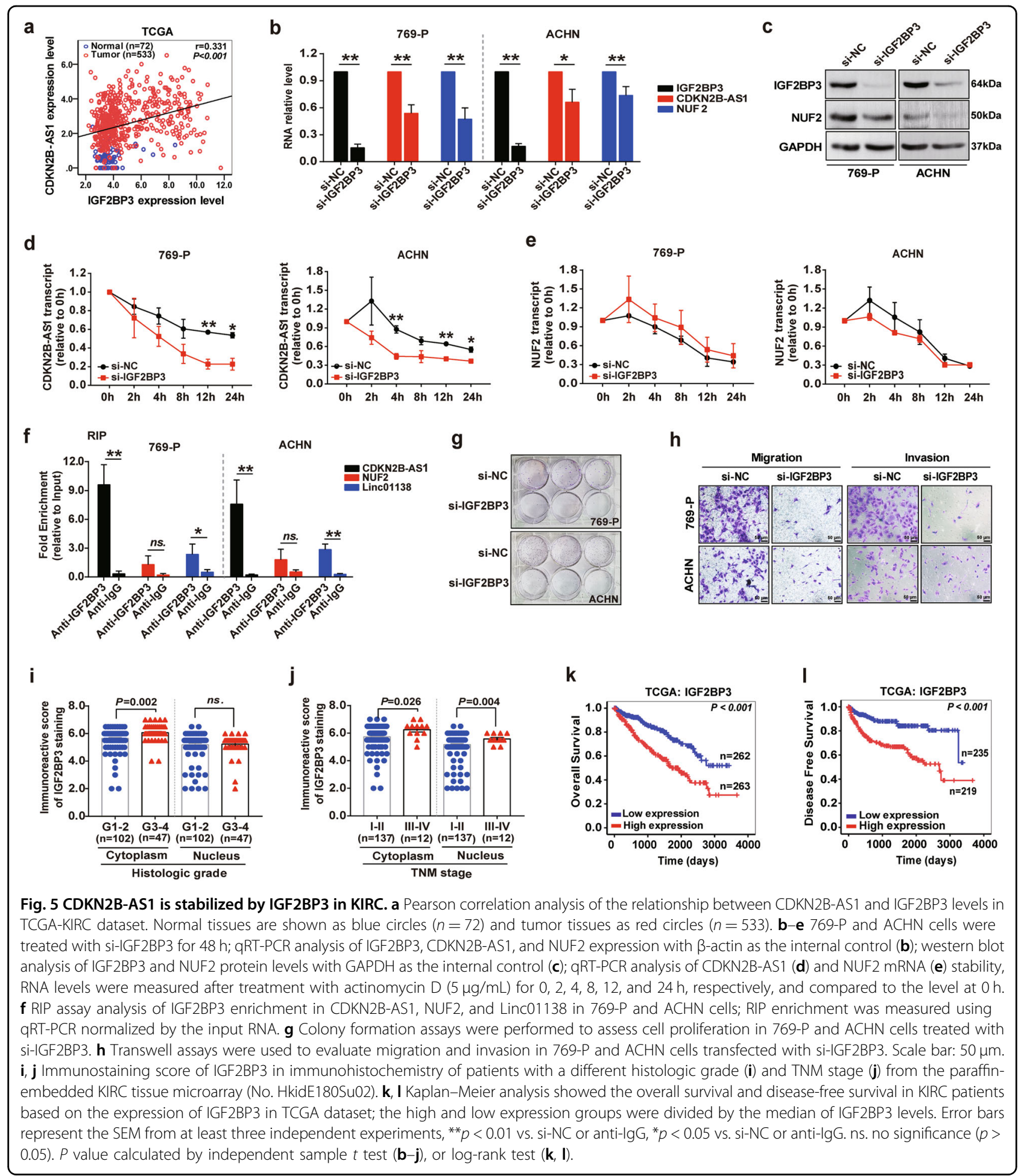

effects of CDKN2B-AS1 on KIRC carcinogenesis in vivo and confirmed its prognostic capability by multivariate analysis. Hence, our findings, together with those of other studies $^{22,23}$, indicate that CDKN2B-AS1 drives the malignancy of KIRC in vitro and in vivo, and has the potential to serve as an independent prognostic factor for worse KIRC progression.

CDKN2B-AS1 may drive cancer progression through several mechanisms. Canonical CDKN2B-AS1 transcriptional mechanisms may play a role, such as the in cis 

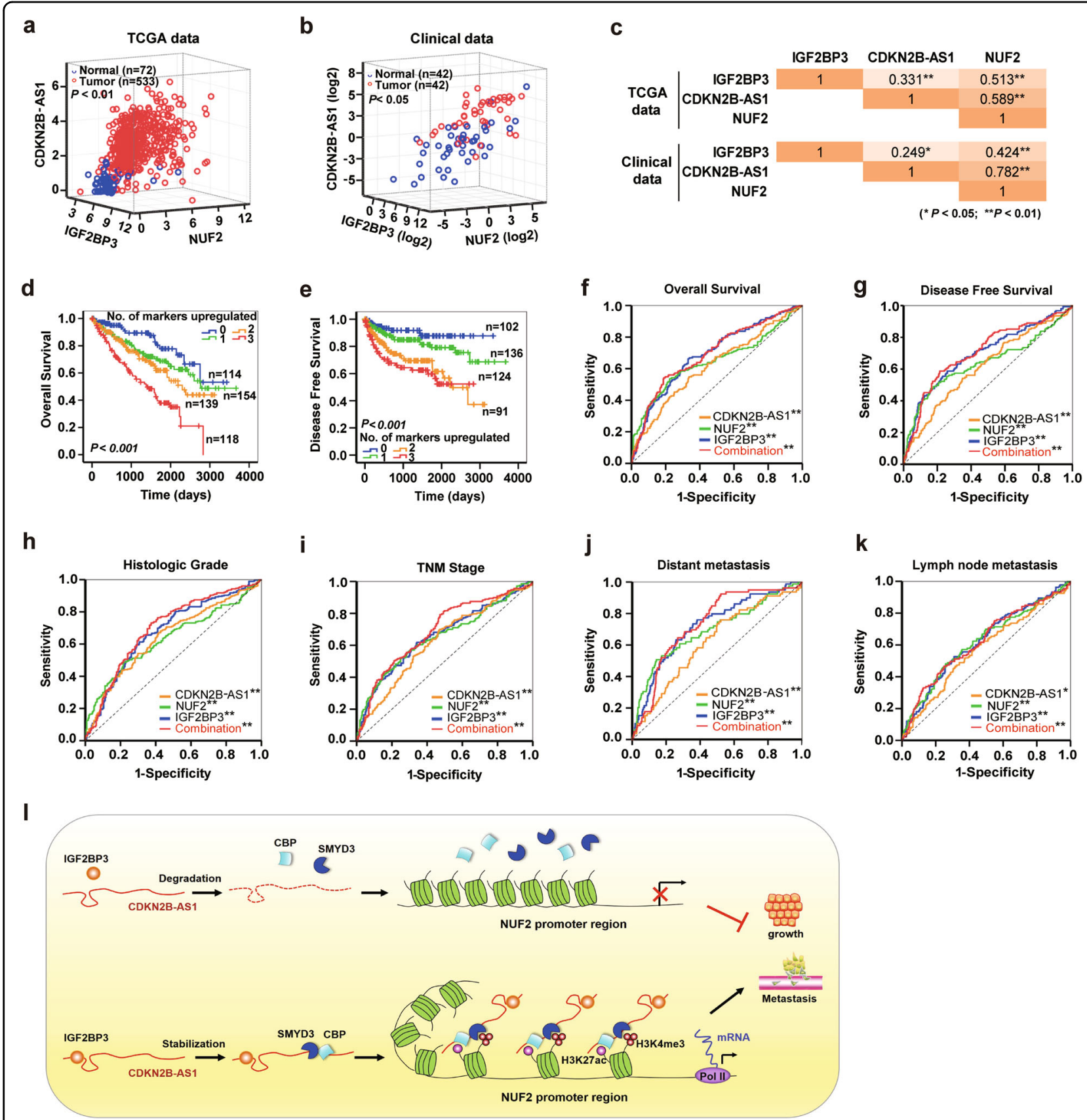

Fig. 6 IGF2BP3/CDKN2B-AS1/NUF2 axis is an attractive candidate as a prognostic and diagnostic biomarker of KIRC. a-c Pearson correlation analysis of the relationship between CDKN2B-AS1, IGF2BP3, and NUF2 levels in TCGA-KIRC dataset (a) and 42 paired KIRC tissues and adjacent normal tissues (b), normal tissues are shown as blue circles and tumor tissues as red circles; the correlation coefficient between each two markers is shown in c. $\mathbf{d}$, e Kaplan-Meier analysis of overall survival and disease-free survival for KIRC patients in TCGA dataset based on the number of upregulated molecular markers; CDKN2B-AS1, NUF2, and IGF2BP3 expression was stratified by the individual medians by RNA-seq data, and the patients were divided into four groups as indicated. $\mathbf{f}, \mathbf{g}$ ROC curve analysis of overall survival (f) and disease-free survival (g) for CDKN2B-AS1, NUF2, and IGF2BP3 as individual biomarkers or as a combined panel (containing CDKN2B-AS1, NUF2, and IGF2BP3). h-k ROC curve analysis for CDKN2B-AS1, NUF2, and IGF2BP3 as individual biomarkers or as a combined panel to discriminate KIRC patients with high tumor grades (G3 and G4) from those with low tumor grades (G1 and G2) (h); KIRC patients with stages III and IV from those with stages I and II (i); KIRC patients with distant metastasis from patients without distant metastasis $(\mathbf{j})$, and KIRC patients with lymph node metastasis from patients without lymph node metastasis $(\mathbf{k}) .{ }^{* *} p<0.01,{ }^{*} p<0.05$. I Schematic diagram of the IGF2BP3/CDKN2B-AS1/NUF2 axis regulating tumor growth and metastasis in KIRC cells. CDKN2B-AS1 is stabilized by specifically binding to IGF2BP3 and acts as a modular scaffold of the CBP and SMYD3 epigenetic-modifying complex to increase H3K27ac and H3K4me3 modification in the promoter region of NUF2, thereby enhancing NUF2 transcription, and promoting tumor cell growth and metastasis. 
Table 1 Univariate and multivariate Cox regression analyses of upregulated marker number for overall survival in KIRC (TCGA dataset).

\begin{tabular}{|c|c|c|c|c|c|c|}
\hline \multirow[t]{2}{*}{ Variables } & \multicolumn{3}{|c|}{ Univariate analysis } & \multicolumn{3}{|c|}{ Multivariate analysis } \\
\hline & HR & $95 \% \mathrm{Cl}$ & $P$ value & HR & $95 \% \mathrm{Cl}$ & $P$ value \\
\hline Sex (male vs. female) & 1.052 & $(0.768,1.442)$ & 0.752 & & & \\
\hline Age ( $>60$ years vs. $\leq 60$ years) & 1.742 & $(1.271,2.386)$ & 0.001 & 1.647 & $(1.183,2.292)$ & 0.003 \\
\hline Histologic grades (G1-G4) & 2.398 & $(1.940,2.964)$ & $<0.001$ & 1.41 & $(1.091,1.822)$ & 0.009 \\
\hline TNM stages (I-IV) & 1.953 & $(1.707,2.236)$ & $<0.001$ & & & \\
\hline Tumor invasions (T1-T4) & 1.992 & $(1.685,2.355)$ & $<0.001$ & & & \\
\hline Distant metastasis (yes vs. no) & 4.544 & $(3.303,6.251)$ & $<0.001$ & & & \\
\hline Lymph node metastasis (yes vs. no) & 1.385 & $(1.000,1.918)$ & 0.05 & & & \\
\hline Tumor size ( $>1.5 \mathrm{~cm}$ vs. $\leq 1.5 \mathrm{~cm}$ ) & 1.675 & $(1.213,2.312)$ & 0.002 & & & \\
\hline Number of upregulated markers (0-3) & 1.585 & $(1.359,1.849)$ & $<0.001$ & 1.22 & $(1.025,1.453)$ & 0.025 \\
\hline
\end{tabular}

HR hazard ratio, Cl confidence interval, TNM tumor node metastasis.

suppression of tumor suppressor genes CDKN2A and

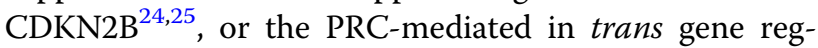
ulation $^{18,26}$. In addition, evidence suggests that CDKN2BAS1 acts as competing endogenous RNA (ceRNA) and as a molecular sponge for microRNA let-7a $\mathrm{a}^{27,28}$, miR$125 \mathrm{a}^{29}$, miR-99a/miR-449a $\mathrm{a}^{30}$, and miR-122-5 $\mathrm{p}^{31}$, which are involved in the progression of prostate cancer, nasopharyngeal cancer, oral carcinoma, gastric cancer, and hepatocellular carcinoma, respectively. Moreover, CDKN2B-AS1 interacts with signaling pathways in cancers, such as p38 MAPK, PI3K/AKT, mTOR, ATM-E2F1, and TGF- $\beta^{19}$. Therefore, it is not surprising that CDKN2B-AS1 can promote RCC carcinogenesis via the $\beta$-catenin pathway or function as a ceRNA for miR-141 to regulate cyclin-D1/D2 expression ${ }^{22,23}$. We used multiple open-source large datasets to identify the potential target genes regulated by $\mathrm{CDKN} 2 \mathrm{~B}-\mathrm{AS} 1$, and also proved in vivo and in vitro that CDKN2B-AS1 affects KIRC tumor growth and metastasis, at least partly through NUF2, which can also serve as an oncogene to promot tumor progression ${ }^{32,33}$. Therefore, our study provides additional mechanistic insight into the role of CDKN2BAS1 in KIRC progression.

The biological function of lncRNAs is largely dependent on their subcellular localization. For instance, lncRNAs in the cytoplasm can regulate the gene expression at the posttranscriptional level, including by acting as ceRNAs and protecting the target mRNAs from repression ${ }^{34,35}$. However, lncRNAs located in the nucleus can participate in the gene regulation at the chromatin modification and gene transcription levels ${ }^{36}$. Although the latest study ${ }^{23}$ showed that CDKN2B-AS1 can function as a ceRNA to regulate cyclin-D1/D2 expression, resulting in $\mathrm{RCC}$ aggressiveness, by using cell cytoplasm/nuclear fractionation assays, we found that CDKN2B-AS1 is mainly localized in the nucleus, as in previous reports on other tumors $^{30,37,38}$, and indicating that CDKN2B-AS1 might regulate NUF2 expression via a transcriptional mechanism. Since CDKN2B-AS1 was reported to cooperate with chromatin-modifying enzymes $\mathrm{CBX} 7 / \mathrm{PRC} 1$ or SUZ12/PRC2, it affects histone H3K27me3 enrichment in the promoter region, thus activating epigenetic activation or gene silencing ${ }^{37,39}$. As ChIP-seq data and ChIP-qPCR assay showed that gene-activated markers H3K27ac and H3K4me3 were more enriched in the NUF2 promoter region of KIRC, CDKN2B-AS1 may modulate NUF2 by changing the level of histone modification in the promoter region. Following, our experiments demonstrated that CDKN2B-AS1 could enhance the recruitment of the CBP and SMYD3 epigenetic modification complex to the NUF2 promoter region, resulting in elevated levels of histones $\mathrm{H} 3 \mathrm{~K} 27 \mathrm{ac}$ and $\mathrm{H} 3 \mathrm{~K} 4 \mathrm{me}$, thereby stimulating NUF2 expression in KIRC cells. However, unlike in other reports $^{37,39}$, CDKN2B-AS1 did not affect H3K27me3 enrichment in the NUF2 promoter region, suggesting that the regulatory effects of CDKN2B-AS1 on genes have tissue and cell specificity.

Until now, most studies on the mechanism underlying CDKN2B-AS1 regulation have mainly investigated at the transcriptional level. For instance, it has been found that CpG methylation impacts the binding of CTCF, SMAD3/ 4 , and $E R \alpha$ to the promoter region and regulates CDKN2B-AS1 expression ${ }^{19}$. In addition, CDKN2B-AS1 could also be regulated by transcription factors, including E2F1, STAT1, SOX2, SP-1, TET2, and c-MYC ${ }^{19}$. Despite a study ${ }^{40}$ in 2017, reporting that Kaposi's sarcomaassociated herpesvirus miRNAs, and its latencyassociated proteins vFLIP and vCyclin could affect the 
stability of CDKN2B-AS1, little was known about CDKN2B-AS1 posttranscriptional regulation, especially whether it was regulated by RNA-binding proteins. In this study, we found that the RNA-binding protein IGF2BP3 interacted with and stabilized CDKN2B-AS1, resulting in augmented posttranscriptional CDKN2B-AS1 expression. This is supported by similar reports on IGF2BP3 binding to and improving the stability of target RNAs, such as the lncRNA linc01138, and HMGA2, IGF2, HMGA2, CCND1, MMP9, and CD44 mRNAs ${ }^{21,41}$, and participating in the progression of various tumors ${ }^{20}$.

IGF2BP3, as a member of the IGF2BP family, binds to and influences the localization, transportation, and stability of target RNAs and plays an important role in cell proliferation, migration, invasion, metabolism, and embryonic development ${ }^{20}$. IGF2BP3 is regarded as an oncofetal protein that is abnormally highly expressed in a variety of tumors, such as pancreatic, colorectal, kidney, and oral squamous cell carcinoma ${ }^{41,42}$. In particular, the results of this and other studies ${ }^{43-46}$ showed that IGF2BP3 also stimulated the progression of KIRC and that IGF2BP3 levels in tumor tissues, and peripheral blood could be used as a predictor of KIRC metastasis and clinical prognosis. In addition to a previous report on IGF2BP3 activating the NF- $\mathrm{KB}$ signaling pathway ${ }^{43}$, we also found that IGF2BP3 can mediate the occurrence and development of KIRC by interacting with and stabilizing CDKN2B-AS1, providing a more specific molecular mechanism for the biological role of IGF2BP3 in KIRC and shedding new light on the utility of IGF2BP3 in clinical practice.

In summary, we found that the upregulated IncRNA CDKN2B-AS1 acts as an essential oncogene, promotes tumor malignant progression in vitro and in vivo, and correlates with poor prognosis in KIRC. Mechanistically, CDKN2B-AS1 was stabilized by interaction with IGF2BP3, and exerted its oncogenic activity by acting as a modular scaffold of the CBP and SMYD3 epigeneticmodifying complex to the promoter region of NUF2, where it enhanced NUF2 transcription by increasing local H3K27ac and H3K4me3 modifications. Clinically, the IGF2BP3/CDKN2B-AS1/NUF2 axis might be an ideal therapeutic target and a promising prognostic and diagnostic indicator for KIRC. Therefore, uncovering the precise role of this regulatory axis in KIRC progression will not only increase our knowledge of lncRNA-regulated therapeutic effects in cancer and their underlying mechanisms, but also help to develop more efficient strategies to treat KIRC.

\footnotetext{
Author details

'Guangdong Key Laboratory of Systems Biology and Synthetic Biology for Urogenital Tumors, Shenzhen Second People's Hospital, First Affiliated Hospital of Shenzhen University, 518000 Shenzhen, Guangdong, China. ${ }^{2}$ Department of Minimally Invasive Intervention, Peking University Shenzhen Hospital, 518000
}

Shenzhen, Guangdong, China. ${ }^{3}$ Department of Otolaryngology, Shenzhen Second People's Hospital, First Affiliated Hospital of Shenzhen University, 518000 Shenzhen, Guangdong, China. ${ }^{4}$ Department of Urology, Longgang District Central Hospital, 518100 Shenzhen, Guangdong, China. ${ }^{5}$ Department of Community Surveillance, The First Affiliated Hospital of Shantou University Medical College, 515041 Shantou, Guangdong, China

\section{Author contributions}

Xina Xie, Aifa Tang and Zesong Li conceived and designed the experiments; Jiatian Lin performed bioinformatical analysis; Xina Xie, Jiatian Lin, Xiaoqin Fan, Yuantang Zhong, Kaiqing Liu, Yonggang Ren, Yequn Chen and Xiangling Chen performed the experiments; Yequn Chen contributed to obtaining the patient tissue samples; Xina Xie and Xiaogin Fan analyzed the data; Daihuan Lai and Xuyi Li contributed reagents and materials; Aifa Tang and Zesong Li supervised the study and wrote the manuscript together with Xina Xie. All authors read and approved the final manuscript.

\section{Conflict of interest}

The authors declare no competing interests.

\section{Ethics statement}

The study was conducted in accordance with the Declaration of Helsinki, and the protocol was approved by the Ethics Committee of the First Affiliated Hospital of Shantou University Medical College (number: 2020-031). All subjects provided informed consent prior to using the tissues samples for scientific research.

\section{Funding}

This study is supported by National Science Foundation of China (81772736, 81902777), the Shenzhen Science and Technology Project

(JCYJ20180228163436705), and the Sanming Project of Medicine in Shenzhen (SZSM201612071).

\section{Publisher's note}

Springer Nature remains neutral with regard to jurisdictional claims in published maps and institutional affiliations.

Supplementary information The online version contains supplementary material available at https://doi.org/10.1038/s41419-021-03489-y.

Received: 5 December 2020 Revised: 27 January 2021 Accepted: 28 January 2021

Published online: 19 February 2021

\footnotetext{
References

1. Jemal, A. et al. Global cancer statistics. CA Cancer J. Clin. 61, 69-90 (2011).

2. Siegel, R. L., Miller, K. D. \& Jemal, A. Cancer statistics, 2020. CA Cancer J. Clin. 70, 7-30 (2020).

3. Zisman, A. et al. Risk group assessment and clinical outcome algorithm to predict the natural history of patients with surgically resected renal cell carcinoma. J. Clin. Oncol. 20, 4559-4566 (2002).

4. Powles, T. et al. European association of urology guidelines for clear cell renal cancers that are resistant to vascular endothelial growth factor receptortargeted therapy. Eur. Urol. 70, 705-706 (2016).

5. Motzer, R. J. \& Molina, A. M. Targeting renal cell carcinoma. J. Clin. Oncol. 27, 3274-3276 (2009).

6. Zhou, J. et al. Loss of DAB2IP in RCC cells enhances their growth and resistance to mTOR-targeted therapies. Oncogene 35, 4663-4674 (2016).

7. McDermott, D. F. et al. Randomized phase III trial of high-dose interleukin-2 versus subcutaneous interleukin-2 and interferon in patients with metastatic renal cell carcinoma. J. Clin. Oncol. 23, 133-141 (2005).

8. Chen, W. et al. Targeting renal cell carcinoma with a HIF-2 antagonist. Nature 539, 112-117 (2016).

9. Yang, J. Patisiran for the treatment of hereditary transthyretin-mediated amyloidosis. Expert Rev. Clin. Pharm. 12, 95-99 (2019).

10. Ponting, C. P., Oliver, P. L. \& Reik, W. Evolution and functions of long noncoding RNAs. Cell 136, 629-641 (2009).
} 
11. Maass, P. G., Luft, F. C. \& Bahring, S. Long non-coding RNA in health and disease. J. Mol. Med. 92, 337-346 (2014).

12. Balas, M. M. \& Johnson, A. M. Exploring the mechanisms behind long noncoding RNAs and cancer. Noncoding RNA Res. 3, 108-117 (2018).

13. Wu, S. M. et al. circlncRNAnet: an integrated web-based resource for mapping functional networks of long or circular forms of noncoding RNAs. Gigascience 7, 1-10 (2018).

14. Li, J. et al. TANRIC: an interactive open platform to explore the function of IncRNAs in cancer. Cancer Res. 75, 3728-3737 (2015).

15. Li, Y. et al. LncMAP: pan-cancer atlas of long noncoding RNA-mediated transcriptional network perturbations. Nucleic Acids Res. 46, 1113-1123 (2018).

16. Schmittgen, T. D. \& Livak, K. J. Analyzing real-time PCR data by the comparative C(T) method. Nat. Protoc. 3, 1101-1108 (2008).

17. Xie, X. et al. A novel IncRNA NR4A1AS up-regulates orphan nuclear receptor NR4A1 expression by blocking UPF1-mediated mRNA destabilization in colorectal cancer. Clin. Sci. 133, 1457-1473 (2019).

18. Huang, M. D. et al. Long non-coding RNA ANRIL is upregulated in hepatocellular carcinoma and regulates cell apoptosis by epigenetic silencing of KLF2. J. Hematol. Oncol. 8, 50 (2015).

19. Kong, Y., Hsieh, C. H. \& Alonso, L. C. ANRIL: A IncRNA at the CDKN2A/B locus with roles in cancer and metabolic disease. Front. Endocrinol. 9, 405 (2018).

20. Bell, J. L. et al. Insulin-like growth factor 2 mRNA-binding proteins (IGF2BPs): post-transcriptional drivers of cancer progression? Cell Mol. Life Sci. 70, 2657-2675 (2013).

21. Li, Z. et al. The LINC01138 drives malignancies via activating arginine methyltransferase 5 in hepatocellular carcinoma. Nat. Commun. 9, 1572 (2018).

22. Li, Q. et al. Highly expressed antisense noncoding RNA in the INK4 locus promotes growth and invasion of renal clear carcinoma cells via the betacatenin pathway. Oncol. Res. 25, 1373-1382 (2017).

23. Dasgupta, P. et al. LncRNA CDKN2B-AS1/miR-141/cyclin D network regulates tumor progression and metastasis of renal cell carcinoma. Cell Death Dis. 11, 660 (2020).

24. Qiu, J. J. et al. The long non-coding RNA ANRIL promotes proliferation and cell cycle progression and inhibits apoptosis and senescence in epithelial ovarian cancer. Oncotarget 7, 32478-32492 (2016).

25. $\mathrm{Xu}, \mathrm{S}$. et al. ANRIL IncRNA triggers efficient therapeutic efficacy by reprogramming the aberrant INK4-hub in melanoma. Cancer Lett. 381, 41-48 (2016).

26. Nie, F. Q. et al. Long noncoding RNA ANRIL promotes non-small cell lung cancer cell proliferation and inhibits apoptosis by silencing KLF2 and P21 expression. Mol. Cancer Ther. 14, 268-277 (2015).

27. Zhao, B. et al. Overexpression of IncRNA ANRIL promoted the proliferation and migration of prostate cancer cells via regulating let-7a/TGF-beta1/ Smad signaling pathway. Cancer Biomark. 21, 613-620 (2018).

28. Wang, Y., Cheng, N. \& Luo, J. Downregulation of IncRNA ANRIL represses tumorigenicity and enhances cisplatin-induced cytotoxicity via regulating microRNA let-7a in nasopharyngeal carcinoma. J. Biochem. Mol. Toxicol. 31, e21904 (2017)
29. Chai, L., Yuan, Y., Chen, C., Zhou, J. \& Wu, Y. The role of long non-coding RNA ANRIL in the carcinogenesis of oral cancer by targeting miR-125a. Biomed. Pharmacother. 103, 38-45 (2018).

30. Zhang, E. B. et al. Long noncoding RNA ANRIL indicates a poor prognosis of gastric cancer and promotes tumor growth by epigenetically silencing of miR99a/miR-449a. Oncotarget 5, 2276-2292 (2014).

31. Ma, J., Li, T., Han, X. \& Yuan, H. Knockdown of LncRNA ANRIL suppresses cell proliferation, metastasis, and invasion via regulating miR-122-5p expression in hepatocellular carcinoma. J. Cancer Res. Clin. Oncol. 144, 205-214 (2018).

32. Kaneko, N. et al. siRNA-mediated knockdown against CDCA1 and KNTC2, both frequently overexpressed in colorectal and gastric cancers, suppresses cell proliferation and induces apoptosis. Biochem. Biophys. Res. Commun. 390, 1235-1240 (2009).

33. Fu, H. L. \& Shao, L. Silencing of NUF2 inhibits proliferation of human osteosarcoma Saos-2 cells. Eur. Rev. Med. Pharm. Sci. 20, 1071-1079 (2016).

34. Cesana, M. et al. A long noncoding RNA controls muscle differentiation by functioning as a competing endogenous RNA. Cell 147, 358-369 (2011).

35. Yuan, J. H. et al. The MBNL3 splicing factor promotes hepatocellular carcinoma by increasing PXN expression through the alternative splicing of IncRNA-PXNAS1. Nat. Cell Biol. 19, 820-832 (2017).

36. Rinn, J. L. \& Chang, H. Y. Genome regulation by long noncoding RNAs. Annu. Rev. Biochem. 81, 145-166 (2012).

37. Yap, K. L. et al. Molecular interplay of the noncoding RNA ANRIL and methylated histone $\mathrm{H} 3$ lysine 27 by polycomb CBX7 in transcriptional silencing of INK4a. Mol. Cell 38, 662-674 (2010).

38. Hoffmann, M. J. et al. Truncated isoforms of IncRNA ANRIL are overexpressed in bladder cancer, but do not contribute to repression of INK4 tumor suppressors. Noncoding RNA 1, 266-284 (2015).

39. Kotake, Y. et al. Long non-coding RNA ANRIL is required for the PRC2 recruitment to and silencing of p15(INK4B) tumor suppressor gene. Oncogene 30, 1956-1962 (2011).

40. Sethuraman, S., Gay, L. A., Jain, V., Haecker, I. \& Renne, R. microRNA dependent and independent deregulation of long non-coding RNAs by an oncogenic herpesvirus. PLoS Pathog. 13, e1006508 (2017).

41. Lederer, M., Bley, N., Schleifer, C. \& Huttelmaier, S. The role of the oncofetal IGF2 mRNA-binding protein 3 (IGF2BP3) in cancer. Semin. Cancer Biol. 29, 3-12 (2014).

42. $\mathrm{Xu}, \mathrm{W}$. et al. Increased IGF2BP3 expression promotes the aggressive phenotypes of colorectal cancer cells in vitro and vivo. J. Cell Physiol. 234, 18466-18479 (2019).

43. Pei, X. et al. Enhanced IMP3 expression activates NF-small ka, CyrillicB pathway and promotes renal cell carcinoma progression. PLOS ONE 10, e0124338 (2015).

44. Tschirdewahn, S. et al. Circulating and tissue IMP3 levels are correlated with poor survival in renal cell carcinoma. Int. J. Cancer 145, 531-539 (2019).

45. Hoffmann, N. E. et al. External validation of IMP3 expression as an independent prognostic marker for metastatic progression and death for patients with clear cell renal cell carcinoma. Cancer 112, 1471-1479 (2008).

46. Jiang, Z. et al. Analysis of RNA-binding protein IMP3 to predict metastasis and prognosis of renal-cell carcinoma: a retrospective study. Lancet Oncol. 7, 556-564 (2006). 